\title{
Perinatal outcome in preterm premature rupture of membranes before 37 weeks of gestation
}

\author{
Malgorzata Swiatkowska-Freund, Anetta Traczyk-Los, Anna Partyka, Kamil Obara, \\ Altankhorol Damdinsuren, Krzysztof Preis
}

Department of Obstetrics, Medical University of Gdansk, Poland

\begin{abstract}
Objectives: The aim of this study was to assess the maternal and neonatal outcome in patients with preterm premature rupture of membranes between 22 to 37 weeks of gestation in comparison to preterm birth patients.

Material and methods: Group of PPROM patients consisted of 127 women, the control group counted 141 women who delivered prematurely. The control group was formed by matching patient with the same gestational age at delivery and neonatal birth weight to every woman from study group. In both groups speculum and ultrasound examinations were performed, microbiological swabs were taken. In unclear cases of PPROM tests detecting amniotic proteins, such as PAMG-1 or IGFBP-1, were performed. According to gestational age at delivery, neonates were divided into subgroups: extremely premature infants ( $<27$ weeks 6 days), moderate premature infants (from 28 weeks 0 days to 33 weeks 6 days), late premature infants (from 34 weeks 0 days to 37 weeks 0 days).

Results: In the study group, median gestational age of delivery was 34 weeks 1 day and the same in control group - 34 weeks and 5 days $(p>0.05)$. Parameters of inflammatory status were more often reported in the PPROM group than in the preterm birth group, even if they weren't statistically significant (positive culture of cervical swab, increased leukocytosis, CRP above 5). The rate of neonate survival was similar in both groups ( $93.7 \%$ and $94.1 \%$ ). Congenital infection was more often diagnosed in group of neonates from PPROM pregnancies than in neonates from control group; ( $36 \%$ and $21.2 \%$ respectively; $p=0.009$ ).

Conclusions: Our research appears to be consistent with theory of inflammatory etiology of PPROM. Optimal management of infection in PPROM patients seems to be the most important in efforts to prolong pregnancy.
\end{abstract}

Key words: 1 preterm delivery; 2 preterm premature rupture of membranes; 3 neonatology autcome

Ginekologia Polska 2019; 90, 11: 645-650

\section{INTRODUCTION}

Preterm rupture of membranes (PROM) complicates approximately $2-5 \%$ of pregnancies before term and $8 \%$ of term gestations [1]. At term it does not significantly influence pregnancy outcome. If it happens before 37 weeks, we describe it as preterm premature rupture of membranes (PPROM) and it increases the risk of preterm labor, intrauterine infection, fetal hypoxia and intrauterine death [1,2]. Pregnancy outcome in cases of spontaneous PPROM depends on gestational age. Approximately $50 \%$ of patients deliver within a week after PPROM, latency in the other half depends on gestational age, fetal condition and presence of infection [3].

Depending on the gestational age, fetal and maternal condition, there is a possibility of conservative management, including steroids administration, antibiotics and magnesium sulfate or active labor induction after steroids administration [4]. Tocolytics are usually not recommended because uterine contractions are usually correlated to intrauterine infection and prolongation of pregnancy in these cases does not decrease the mortality and morbidity of women and children $[5,6]$.

Lack of amniotic fluid in the uterus, especially before the $24^{\text {th }}$ week of gestation, disturbs fetal lung development. Alveoli do not form without amniotic fluid even after steroids administration. Respiratory distress in children born after PPROM, after they had spent some time in uterus without amniotic fluid, is often more severe than we expect. Neonates have also higher risk of severe inborn infection. Both factors - respiratory distress and infection - worsen prognosis for the children after PPROM $[7,8]$.

There are only a few trials trying to compare different approaches to ahydramnios caused by PPROM. They 
concentrate on two topics: sealing the hole in membranes (amniopatch, immunologic sealants, membranes) and supplementing amniotic fluid (amnioinfusion). As of now, there is no answer to if any type of treatment improves pregnancy outcome $[9,10]$.

\section{Objectives}

Authors compared pregnancy outcome in patients who delivered before term with and without PPROM.

\section{MATERIAL AND METHODS}

The study group consisted of 127 women who were admitted to the Department of Obstetrics of Medical University from October 2009 to 2014 with preterm premature rupture of membranes (between 22 and 37 weeks of gestation).The control group (141 women) was formed by matching patient with the same gestational age at delivery and neonatal birth weight to every woman from study group. According to gestational age at delivery, neonates were divided into subgroups: extremely premature infants $(<27$ weeks 6 days), moderate premature infants (from 28 weeks 0 days to 33 weeks 6 days), late premature infants (from 34 weeks 6 days to 37 weeks 0 days).

To confirm PPROM, sterile speculum examinations were performed with the visualization of amniotic fluid leaking from the cervical canal. In unclear cases tests detecting amniotic proteins, such as PAMG-1 (Placental alpha microglobulin-1) or IGFBP-1 (Insulin -like growth factor binding protein 1), were performed. Ultrasound examinations were performed in every patient - fetal well-being, amniotic fluid index and signs of placental abruption were determined. The exclusion criteria included: multiple pregnancy, intrauterine fetal death, fetal congenital malformations, previous cervical surgery and uncertain diagnosis of PPROM.

According to our Department regulation, patients with PPROM before 34 weeks 0 days of gestation were managed expectantly if no maternal or fetal contraindication were present. Induction of labor wasn't offered to these patients unless intrauterine infection was diagnosed. Tocolysis wasn't ordered to pregnant patients with PPROM. A single course of corticosteroids was offered to every pregnant woman between 24 weeks 0 days and 34 weeks 6 days. Prophylactic antibiotics were given to patients in the latent phase of PPROM from 24 weeks 0 days to 33 weeks 6 days. Every week patients were examined vaginally (speculum examination) and had cervical swabs taken. If a microbiological swab was positive, antibiotics were administered. Similarly, CRP and leukocytosis were evaluated every week. Fetal status was assessed every seven days by ultrasound examination. In case of patients with PPROM after 34 weeks 0 days of gestation, who didn't have contraindication to labor, delivery was induced, mostly with Oxytocin infusion (usually after
12 hours of expectancy for spontaneous contractions). Neither tocolysis nor corticosteroids were offered in this group of patients. Maternal infusion of magnesium sulfate as fetal neuroprotection wasn't recommended for patients before 32 weeks 0 days.

Data from medical records were analyzed to compare pregnancy complications and outcome in the study group and control group. Information regarding gestational age at PPROM, laboratory symptoms of infection (CRP > 5, leukocytosis $>15000 / \mathrm{dL}$, positive culture of cervical swab), steroids and antibiotics administration, gestational age at delivery, mode of delivery, indication for cesarean section, birth weight of neonate, respiratory distress syndrome (type of assisted ventilation), leukocytosis and CRP in neonate, results of microbiological tests in neonates, length of hospital stay of neonate was collected. Congenital infection was diagnosed based on a minimum of two signs of infection in clinical examination and/or increased values of inflammatory parameters in neonate. Clinical symptoms of congenital infection were defined as breathing disturbances (tachypnea, apnea), tachycardia, bradycardia, significant variability of heart rate, emesis, hypotonia, muscle tremors, convulsions, instability of body temperature, lethargy, circulation disturbances, and metabolic disturbances.

The normality of the layout of continuous variables was assessed by means of the Shapiro-Wilk test. Statistical characteristics of continuous variables were presented by means of arithmetic means, standard deviations, medians as well as minimal and maximal values. For inter-group comparisons Student's t-test for non-linked variables or U Mann-Whitney test were applied. In order to compare the layouts of discrete variables, Pearson's chi-squared test or Fisher's exact test were used. All calculations were carried out by means of Statistica10 software (StatSoft, the USA), where value $p<0.05$ was accepted as a statistically significant.

\section{RESULTS}

Average patient age at admission to hospital was $30.2 \pm 5.4$ years in the study group and $29.8 \pm 4.8$ years in the control group ( $p>0.05$ ). As far as gravidity was concerned, $48 \%$ of the patients in study group and $43.3 \%$ of the patients in control group were nulliparous, while $52 \%$ and $56.7 \%$ had had at least one delivery ( $p>0.05)$. In the study group of 127 women median gestational age of delivery was 34 weeks 1 day ( 23 weeks and 5 days to 37 weeks 0 days) and the same in the control group -34 weeks and 5 days, from 22 weeks and 4 days to 37 weeks and 0 days $(p>0.05)$. Median gestational age of delivery in subgroups was: before 27 weeks and 6 day -26 weeks and 0 days in PPROM group and 25 weeks 5 days in control group ( $p>0.05$ ), from 28 weeks and 0 days to 33 weeks and 6 days -31 weeks and 4 days in both groups $(p>0.05)$ and from 
34 weeks and 0 days to 37 weeks and 0 days -35 weeks and 3 days in PPROM group and 35 weeks 4 days in control group ( $\mathrm{p}<0.05)$.

The median gestational age of PPROM in the study group was 33 weeks 5 days (from 21 weeks and 4 days to 36 weeks and 6 days). In details: in the group of patients who delivered before 27 weeks 6 days -24 weeks 6 days, from 28 weeks 0 days to 33 weeks 6 days -30 weeks 1 day, from 34 weeks 0 days to 37 weeks 0 days -35 weeks 2 days. The median time from PPROM to delivery was 1 day ( 0 to 71 days); 9 days in patients, who delivered before 28 weeks of gestation; 11 days in patients who delivered from 28 weeks and 0 days to 33 weeks and 6 days and 1 day in patients who delivered after 33 weeks and 6 days. In the analyzed group 42 patients (33\%) delivered on the day, when PPROM occurred, while 85 (66.9\%) delivered later. Among 42 pregnant women who delivered on the day of PPROM, gestational age of 36 (85.7\%) of them was greater than 33 weeks 6 days of gestation, when in 6 (14.3\%) of them was between 28 weeks 0 days and 33 weeks 6 days. None of PPROM patients who delivered before 28 weeks of pregnancy delivered on the day when PPROM occurred.

Positive culture of cervical swabs were noted in 62 patients with PPROM (49\%) and in 51 patients in the control group (36\%) $(p>0.05, p=0.101)$; in 3 patients $(27 \%)$, who delivered before 28 weeks of gestation in the PPROM group and in 5 patients $(50 \%)$ in the control group ( $p>0.05)$; in 27 patients (56\%), who delivered from 28 weeks 0 days to 33 weeks 6 days in the PPROM group and in 16 patients $(36 \%)$ in the control group ( $p=0.06$ ); in the group of patients who delivered from 34 weeks 0 days to 37 weeks 0 days - in 32 pregnant in the PPROM group (47\%) and in 30 patients $(35 \%)$ in the control group $(p<0.05)$. Increased leukocytosis was noted at least once during latent period in 65 women with PPROM (51.2\%), in the control group leukocytosis greater than 15000 was found in 43 patients (30.7\%) $(\mathrm{p}<0.001)$. In details, in the study and in the control group in 6 patients (54\%) versus 3 patients (33\%) who delivered before 28 weeks of pregnancy, in 30 patients (62\%) versus 18 patients (38\%) who delivered from $28 \mathrm{w} 0 \mathrm{~d}$ to $33 \mathrm{w} 6 \mathrm{~d}$ and in 29 patients (43\%) versus 22 patients (27\%) who delivered later ( $p>0.05 ; p<0.05 ; p<0.05$; respectively) increased leukocytosis was noted. CRP above 5 was observed in 77 patients (68.7\%) in the study group versus 29 patients (56.9\%) in the control group ( $p<0.001$ and $p>0.05$ ). CRP wasn't evaluated in 15 patients (12\%) from the study group and in 90 patients (63.8\%) from the control group.

During latent time prophylactic antibiotics were used in 73 patients $(57.5 \%)$ in the group of PPROM patients. Additionally, antibiotics were administered if increased CRP, leukocytosis or positive microbiological swab was observed. To conclude, antibiotics were given to 99 patients in the
PPROM group before delivery (78\%), while in the control group 50 patients $(35.5 \%)(p<0.001)$.

Steroids were administered in 66 patients before 34 weeks 6 days in the study group (52\%), and in 49 patients in the control group (35\%) $(p<0.05)$; in a group of women who delivered before 28 w0d in $10(90 \%)$ in the PPROM group and in 7 (70\%) in the preterm delivery group; in a group of patients who delivered from $28 \mathrm{w} 0 \mathrm{~d}$ to $33 \mathrm{w} 6 \mathrm{~d}$ in $41(85 \%)$ in the study group and in $27(56 \%)$ in the control group. Patients who delivered after $33 \mathrm{w} 6 \mathrm{~d}$ received steroids in $22 \%$ (15 pregnant) in the PPROM group and in $18 \%$ (15 pregnant) in the control group $(p>0.05, p<0.05$, $\mathrm{p}>0.05$; respectively).

In the study group 77 patients (61\%) delivered vaginally - 73\% (8 pregnant women) before 28 w0d, 59\% (28 pregnant) from $28 w 0 d$ to $33 w 6 d$ and $60 \%$ (41 pregnant women) from $34 w 0 d$ to $37 w 0 d$. In the remaining 50 women (39\%), cesarean sections were performed. In the control group, 38 patients delivered vaginally $(27 \%)-30 \%$ (3 pregnant women) before $28 w 0 d, 29 \%$ (13 pregnant women) from $28 w 0 d$ to $33 w 6 d$ and $27 \%$ (22 pregnant women) from $34 w 0 d$ to $37 w 0 d$. Cesarean sections were performed in 103 patients (73\%). The cesarean section rate was higher in the control group $(p<0.001)$ and in the subgroups this trend was also observed $(p=0.05, p<0.05, p<0.05)$.

The cesarean section in the study group was performed most frequently due to fetal distress ( 28 women - $58.3 \%$ ) or intrauterine infection ( 10 women $-20.8 \%$ ), but also due to fetal malpresentation ( 9 women - 18.8\%), placental abruption ( 2 women $-4.2 \%)$, preeclampsia ( 1 woman $-2.1 \%$ ), threatening rupture of uterus ( 4 women $-8.3 \%$ ) and maternal indications ( 8 women - 16.7\%), fetal indications (1 woman - 2.1\%). Indications for cesarean sections in the control group was mostly fetal distress (66 women - 64.7\%). Other indications were: intrauterine infection ( 3 women $-2.9 \%$ ), fetal malpresentation (8 women $-7.8 \%$ ), placental abruption (6 women - 5.9\%), preeclampsia (15 women $-14.7 \%$ ), threatening rupture of uterus ( 4 women $-3.9 \%$ ) and maternal indications (13 women - 12.7\%), fetal indications ( 4 women $-3.9 \%$ ). In three cases, indications for cesarean section were unknown. The group of extremely preterm infants consisted of 11 neonates (9\%) in the PPROM group and 10 neonates (7\%) in the preterm delivery group, moderate preterm infants - 48 (38\%) in the PPROM group and $47(33 \%)$ in the preterm delivery group, late preterm infants - 68 (53\%) in the PPROM group and $84(60 \%)$ in the control group ( $p>0.05, p>0.05, p<0.05$ ).

The average birth weight was similar in the study and the control group (2117.9 $\mathrm{g}$ and $2245.0 \mathrm{~g}$ respectively, $\mathrm{p}>0.05)$. In extremely preterm infants it was $938 \mathrm{~g}$ and $832 \mathrm{~g} \mathrm{(}>0.05)$, in moderate preterm infants $1717 \mathrm{~g}$ and $1766 \mathrm{~g}(\mathrm{p}>0.05)$ and in late preterm infants $2589 \mathrm{~g}$ and 
$2667 \mathrm{~g}(\mathrm{p}>0.05)$ in the PPROM group and the control group respectively.

The rate of neonate survival to discharge was similar in both groups (93.7\% and $94.1 \%$ respectively; $p>0.05$ ). Rates of survival increased with increasing gestational age in both groups; in the PPROM group: 73\% (<28w0d), 94\% (from $28 w 0 d$ to $33 w 6 d$ ), $99 \%$ (from $34 w 0 d$ to $37 w 0 d$ ) and in the control group: $60 \%, 94 \%, 97 \%$ respectively $(p<0.05$, $p>0.05, p>0.05$ ).

Incidence of neonatal respiratory distress syndrome was similar in both groups ( $42.5 \%$ vs $45 \%$; $p>0.05$ ). In extremely preterm infants in $90 \%$ (10 neonates) and in $89 \%$ (8 neonates) ( $p>0.05)$, in moderate preterm infants in 35 (73\%) and in 35 neonates $(74 \%)(p>0.05)$, in late preterm infants in 10 neonates $(15 \%)$ and in 21 neonates $(25 \%)$ ( $p>0.05)$ in the PPROM group and the control group respectively.

Assisted ventilation was used in 48 neonates (38\%) in the study group and in 59 children (42\%) in the control group ( $p>0.05)$; in 10 neonates (90\%) and in 8 neonates ( $80 \%)$ in extremely preterm infants $(p>0.05)$, in 32 neonates $(66 \%)$ and in 34 neonates $(74 \%)$ in moderate preterm infants $(p>0.05)$, in 6 neonates (9\%) and in 17 neonates (20\%) in late preterm infants $(p=0.059)$. Congenital infection was diagnosed more often in the group of neonates from PPROM pregnancies (45 neonates) than in neonates from the control group (29 neonates); ( $36 \%$ and $21.2 \%$ respectively; $p=0.009)$. In detail, infection was present in 6 (55\%) extremely preterm neonates in the PPROM group and in $3(37 \%)$ in the control group $(p>0.05)$, in $25(52 \%)$ moderate preterm neonates in the PPROM group and in $14(31 \%)$ in the control group $(p<0.05)$ and in $13(19 \%)$ late preterm neonates in study group and in $11(13 \%)$ in preterm delivery group ( $p>0.05)$. Differences were also noted regarding leukocytosis in neonate above 15 000/dL (61.5\% and 49.2\% respectively), but it was not statistically significant $(p=0.059)$. In detail, high leukocytosis was found in extremely preterm in $66 \%$ (6 neonates) and $37 \%$ (3 neonates) ( $p>0.05$ ), in moderate preterm infants in $56 \%$ ( 26 neonates) and in $40 \%$ (18 neonates) ( $p>0.05$ ) and in late preterm infants in 60\% (40 neonates) and in 50\% (41 neonates) $(p>0.05)$ in the PPROM group and the control group respectively.

Number of neonates with CRP above 5 was similar in both groups (22.3\% and $24.4 \%$ respectively; $p>0.05)$. In extremely preterm infants CRP was above 5 in 33\% (3 neonates) versus $37 \%$ ( 3 neonates) ( $p>0.05$ ), in moderate preterm infants $38 \%$ ( 17 neonates) versus $35 \%$ ( 15 neonates) ( $p>0.05$ ) and in late preterm infants $12 \%$ ( 8 neonates) versus $17 \%$ (14 neonates) $(p>0.05)$ in the PPROM group and the control group respectively.

Antibiotic therapy was administered in 88 (69\%) of neonates from the study group versus $80(61 \%)$ neonates from the control group ( $p=0.04)$. In extremely preterm infants antibiotics were given to 10 neonates (90\%) and in 9 neonates $(90 \%)(p>0.05)$, in moderate preterm infants in 47 neonates (98\%) and in 44 neonates $(94 \%)(p>0.05)$ and in late preterm infants in 31 neonates (45\%) and in 28 neonates $(34 \%)(p>0.05)$ in the PPROM group and the control group respectively.

Median hospital stay of the neonates in the study group was 11 days ( 0 to 79 days) and in the control group -8 days (0 to 82 days), and the difference wasn't significant ( $p>0.05$ ). In extremely preterm infants it was 67 and 54 days ( $p>0.05$ ), in moderate preterm infants 25 and 30 days $(p>0.05)$ and in late preterm infants 8 and 9 days $(p>0.05)$ in the PPROM group and control group respectively.

\section{DISCUSSION}

According to Romero's definition preterm labor results from "pathological activation" of one or more components of "common pathway of parturition". Uterine myometrial contractility, cervical ripening and membranes rupture, if activated synchronously are said to be the most important factors leading to preterm delivery. Asynchronous activation of one of these components results in cervical insufficiency, preterm uterine contractions or preterm premature rupture of membranes [11, 12]. So far there are many dimensions identified as responsible or connected with mentioned above clinical situations [12]. Prostaglandins and matrix-degrading enzymes (mediators of infection) are considered to be crucial factors in collagen degradation and weakening of membranes, which leads to its rupture [13]. Clinical studies have confirmed infectious theory - bacteria's have the ability to cross even intact membranes and positive amniotic fluid cultures are identified in $12.8 \%$ of patients with preterm birth and $32 \%$ with preterm premature rupture of membranes according to Romero's researches [14]. Caroll S.G. et al. [15] showed that positive culture of swabs from the vagina predicts $53 \%$ of intraamniotic infections. In our study we haven't examined amniotic fluid for presence of microbial invasion, but we also observed positive culture of cervical swab significantly more frequent in group of patients with rupture of membranes than in patients with preterm birth. Similarly, increased leukocytosis and CRP were noted more often in groups of pregnant women with PPROM than in groups of patients with preterm birth. CRP isn't routinely ordered in case of patients with risk of preterm delivery, that's why increased value of this marker was observed only in $13 \%$ of patients among the patients in this group. According to literature neither CRP nor leukocytosis are considered to be highly specific in detecting intrauterine infection and are not even recommended in some countries to be assessed. The estimated sensitivity rate of leukocytosis in detecting chorioamnionitis is $29-47 \%$ [16], when specificity of CRP is 38-55\% [17]. Antibiotics were applied 
in groups of PPROM patients more often than in control groups. Antibiotics weren't offered routinely to everyone. Due to hospital regulation, antibiotics were offered prophylactically or in case of increased inflammatory indicators or symptoms of infection.

Nowadays, according to Polish Society of Gynecologists and Obstetricians the most optimal approach to management of PPROM patients is expectant management with antibiotics therapy to prolong latency of pregnancy. After 34 weeks of pregnancy induction of delivery with oxytocin is indicated only if intrauterine infection is suspected. Regardless of clinical management birth in 7 days following rupture of membranes occurs in $50 \%$ patients [3]. The median length of latency from PPROM to delivery in the examined group was 1 day (from 0 to 71 days). Comparing to other publications, median latency time in the PPROM group was short because in the evaluated group nearly half consisted of patients after 34 weeks of gestation, where due to hospital regulation and ACOG recommendations active management was recommended. In patients with PPROM before 28 weeks of gestation median latency time was 9 days and 11 days if delivery took place between $28+0$ to $33+6$, similar results were presented by A. Peaceman. In a group of 1377 patient's median latency time between 24-28 weeks was approximately 9 days, and between 30-31 weeks was 6 days [18].

The difference in the frequency of cesarean section was observed in the research. This operative procedure was more often performed in group of patients with intact membranes than in PPROM group. The main indication for cesarean section in both groups was fetal distress, while in the group of PPROM patients could have been related to active management (induction of delivery) in case of patients after 34 weeks of gestation. Threatening intrauterine infection was the second main indication for cesarean section in the PPROM group, what is consistent with published data.

Congenital infection was more often detected in all subgroups of neonates from the study group than the control group, what is consistent with fact of higher incidence of increased parameters of inflammation and positive cervical swabs in pregnant with PPROM. As a consequence of this, antibiotics were also more often administered in the study group. Comparable results were published by Moratti [19]. According to data published by Bengtson and Dale [20,21] respiratory distress syndrome incidence in neonates from pregnancies complicated by PPROM is evaluated to be $10-40 \%$. In general, in our study we noticed high ratio of respiratory distress, comparable in both groups of neonates $(42.5 \%$ and $45 \%)$. In case of extremely and moderate preterm neonates, it was a result of early gestational age at delivery and impossibility of steroids administration. In the group of neonates delivered after 34 weeks of gestation, res- piratory distress syndrome was more often observed in the control group, which is probably connected with the high percentage of cesarean section performed in this group.

Considering neonatal survival rate, we haven't found significant difference between both groups of patients. Neonatal death mostly occurred in cases of neonates born before 26 weeks of gestation with low birth weight. Glass estimates that extremely premature infants with extremely low birth weight have $30-50 \%$ risk of mortality and $20-50 \%$ risk of morbidity in survivors [22]. Probably, if patients with PPROM (especially after $28^{\text {th }}$ week of pregnancy) are appropriately covered with antibiotics, which increases the latent time of pregnancy and offers the possibility of steroids and magnesium sulfate administration, the presence of PPROM doesn't influence the rate of mortality in neonates.

\section{CONCLUSIONS}

Our research appears to be consistent with theory of inflammatory etiology of PPROM. Optimal management of infection in PPROM patients seems to be the most important in efforts to prolong latency of pregnancy and decrease mortality and morbidity in neonates.

\section{REFERENCES}

1. Harger JH, Toumala RE, Gibbs RS, et al. Premature rupture of membranes. Obstet Gynecol. 2013; 122: 918-930, doi: 10.1097/01. AOG.0000435415.21944.8f.

2. Getahun D, Strickland D, Ananth CV, et al. Recurrence of preterm premature rupture of membranes in relation to interval between pregnancies. Am J Obstet Gynecol. 2010; 202(6): 570.e1-570.e6, doi: 10.1016/j. ajog.2009.12.010, indexed in Pubmed: 20132922.

3. Mercer BM. Preterm premature rupture of the membranes. Obstet Gynecol. 2003; 101(1): 178-193, doi: 10.1016/s0029-7844(02)02366-9, indexed in Pubmed: 12517665.

4. American College of Obstetricians and Gynecologists' Committee on Practice Bulletins-Obstetrics. Practice Bulletin No. 172: Premature Rupture of Membranes. Obstet Gynecol. 2016; 128(4): e165-e177, doi: 10.1097/AOG.0000000000001712, indexed in Pubmed: 27661655.

5. Jazayeri A, Jazayeri MK, Sutkin G. Tocolysis does not improve neonatal outcome in patients with preterm rupture of membranes. Am J Perinatol. 2003; 20(4): 189-193, doi: 10.1055/s-2003-40606, indexed in Pubmed: 12874729.

6. Ehsanipoor RM, Shrivastava VK, Lee RM, et al. A randomized, double-masked trial of prophylactic indomethacin tocolysis versus placebo in women with premature rupture of membranes. Am J Perinatol. 2011; 28(6): 473-478, doi: 10.1055/s-0030-1270118, indexed in Pubmed: 21170827.

7. WatersTP,Mercer BM. The management of preterm premature rupture of the membranes near the limit of fetal viability. Am J Obstet Gynecol. 2009; 201(3): 230-240, doi: 10.1016/j.ajog.2009.06.049, indexed in Pubmed: 19733274.

8. Winn HN, Chen M, Amon E, et al. Neonatal pulmonary hypoplasia and perinatal mortality in patients with midtrimester rupture of amniotic membranes--a critical analysis. Am J Obstet Gynecol. 2000; 182(6): 16381644, doi: 10.1067/mob.2000.107435, indexed in Pubmed: 10871491.

9. Sciscione AC, Manley JS, Pollock $M$, et al. Intracervical fibrin sealants: a potential treatment for early preterm premature rupture of the membranes. Am J Obstet Gynecol. 2001; 184(3): 368-373, doi: 10.1067/mob.2001.111796, indexed in Pubmed: 11228489.

10. Singla A, Yadav $P$, Vaid NB, et al. Transabdominal amnioinfusion in preterm premature rupture of membranes. Int J Gynaecol Obstet. 2010; 108(3): 199-202, doi: 10.1016/j.ijgo.2009.09.032, indexed in Pubmed: 20015490.

11. Romero R, Gomez R, Mazor M, et al. The preterm labor syndrome. In: Elder MG, Lamont RF. ed. Preterm labor. Churchill Livingstone, New York 1997: 29-49.

12. Gotsch F, Romero R, Erez O, et al. The preterm parturition syndrome and its implications for understanding the biology, risk assessment, diagnosis, treatment 
and prevention of preterm birth.J Matern Fetal Neonatal Med. 2009;22 Suppl 2: 5-23, doi: 10.1080/14767050902860690, indexed in Pubmed: 19951079.

13. Draper $D$, Jones W, Heine RP, et al. Trichomonas vaginalis Weakens human Amniochorion in an in vitro model of premature membrane rupture. Infect Dis Obstet Gynecol. 1995; 2(6): 267-274, doi: 10.1155/S1064744995000160, indexed in Pubmed: 18475407.

14. Romero R, Salafia CM, Athanassiadis AP, et al. The relationship between acute inflammatory lesions of the preterm placenta and amniotic fluid microbiology. Am J Obstet Gynecol. 1992; 166(5): 1382-1388, doi: 10.1016/0002-9378(92)91609-e, indexed in Pubmed: 1595794.

15. Carroll SG, Papaioannou S, Ntumazah IL, et al. Lower genital tract swabs in the prediction of intrauterine infection in preterm prelabour rupture of the membranes. Br J Obstet Gynaecol. 1996; 103(1): 54-59, doi: 10.1111/j.1471-0528.1996.tb09515.x, indexed in Pubmed: 8608099.

16. Ismail MA, Zinaman MJ, Lowensohn RI, et al. The significance of C-reactive protein levels in women with premature rupture of membranes. Am J Obstet Gynecol. 1985; 151(4): 541-544, doi: 10.1016/00029378(85)90285-6, indexed in Pubmed: 3976754.

17. Watts $\mathrm{DH}$, Krohn MA, Hillier SL, et al. Characteristics of women in preterm labor associated with elevated C-reactive protein levels. Obstet Gynecol. 1993; 82(4 Pt 1): 509-514, indexed in Pubmed: 8377973.
18. Peaceman AM, Lai Y, Rouse DJ, et al. Eunice Kennedy Shriver National Institute of Child Health and Human Development Maternal-Fetal Medicine Units Network. Length of latency with preterm premature rupture of membranes before 32 weeks' gestation. Am J Perinatol. 2015; 32(1): 57-62, doi: 10.1055/s-0034-1373846, indexed in Pubmed: 24819145.

19. Moretti M, Sibai BM. Maternal and perinatal outcome of expectant management of premature rupture of membranes in the midtrimester. Am J Obstet Gynecol. 1988; 159(2): 390-396, doi: 10.1016/s00029378(88)80092-9, indexed in Pubmed: 3407697.

20. BENGTSON J, VanMARTER L, BARSS V, et al. Pregnancy Outcome After Premature Rupture of the Membranes at or Before 26 Weeks' Gestation. Am J Obstet Gynecol. 1989; 73(6): 921-927, doi: 10.1097/00006250198906000-00002.

21. Dale PO, Tanbo T, Bendvold E, et al. Duration of the latency period in preterm premature rupture of the membranes. Maternal and neonatal consequences of expectant management. Eur J Obstet Gynecol Reprod Biol. 1989; 30(3): 257-262, doi: 10.1016/0028-2243(89)90010-5, indexed in Pubmed: 2714507.

22. Glass HC, Costarino AT, Stayer SA, et al. Outcomes for extremely premature infants. Anesth Analg. 2015; 120(6): 1337-1351, doi: 10.1213/ANE.0000000000000705, indexed in Pubmed: 25988638. 